\title{
Optimal Medical Therapy of Chronic Stable Angina: Current Guidelines and Future Perspectives
}

\section{Francesco Pelliccia*}

Department of Heart and Great Vessels, Sapienza University, Italy

The optimal medical therapy of patients with chronic stable angina is a controversial issue. While the management of stable angina has certainly improved during the last decade, a number of challenges remain. The European Survey showed that up to 30\% of patients do not receive antiplatelet drugs, $50 \%$ do not receive a beta-blocker, and $25 \%$ do not use a lipid-lowering agent [1]. Furthermore, guidelines for the management of stable angina are based on therapies that, at variance with other diseases, have not been extensively evaluated in large randomized trials. Also, both American and European guidelines have been published years ago, and therefore do not take into consideration the role of newer treatment options in management algorithms. As a consequence, many patients at greatest risk receive less than optimal treatment nowadays.

\section{Current Guidelines}

The trials of medical therapy vs. interventional approaches in stable angina were carried out almost three decades ago, when routine managements differed greatly from those currently adopted. Given that European [2] and American [3] guidelines were published in 2006 and 2007, respectively, review is expecting soon. In 2011, the National Institute for Health and Clinical Excellence (NICE) published the recommendations for the management of stable angina [4]. According to the UK document, optimal medical therapy should comprise one or two anti-anginal agents as needed, without distinguishing between betablockers or calcium channel blockers as first-line anti-anginal therapy. The guidelines recommend switching between these treatments if needed, or combining them if symptoms are not satisfactorily controlled. The major limitations of NICE guidelines are twofold: (i) they equally support the use of beta-blockers and calcium-antagonists, despite the latter have been studied in the INVEST trial only in 2003 [5]; (ii) they allow the newer antianginal drugs, i.e. ivabradine, ranolazine and nicorandil, to be used only when older drugs fail.

\section{The Newer Anti-Anginal Treatments}

There is now evidence to suggest the possibility that the newer antianginal treatments may act as 'disease modifiers.'

Ivabradine acts on the $\mathrm{I}_{\text {fcurrent }}$ which regulates the intrinsic pacemaker activity of the sinoatrial node, resulting in reduction in heart rate and myocardial oxygen demand. Ivabradine has a proven antianginal efficacy and is effective beyond beta-blockers, as it increases coronary blood flow and preserves myocardial contractility and relaxation [6].

Ranolazine inhibits the late sodium influx across the sarcolemma, thereby attenuating abnormalities in ventricular repolarisation and contractility associated with myocardial ischemia, as well as preventing calcium overload that causes cardiac ischemia. Several studies have already demonstrated the antianginal and anti-ischemic activities of ranolazine [7]. At variance with beta-blockers and calcium-antagonists, ranolazine does not significantly alter heart rate or increase systolic blood pressure, thus explaining its relevant role in those with angina refractory to maximal tolerated doses of other anti-anginal medications. In addition, ranolazine has the potential of limiting myocardial ischemia, thus playing a role in the pathophysiology of peri-procedural myocardial infarction during percutaneous coronary intervention. A recent randomized, pilot study has demonstrated that pretreatment with ranolazine $1,000 \mathrm{mg}$ twice daily for 7 days significantly reduced procedural myocardial injury in elective PCI [8].

Nicorandil is a potassium channel activator, as well as acting as a nitrate-like epicardial coronary vasodilator, lowering preload through venodilation. Treatment is associated with protection of the myocardium during ischemia, shortening action potential duration and preventing intracellular calcium toxicity [9].

\section{Future Perspectives}

First-line treatment should not only provide symptomatic relief, but also address the underlying disease mechanism(s). Beta-blockers are here to stay as first-line therapy of stable angina, as they have diseasemodifying properties and exert favourable effects on arrhythmias, heart failure and prevention of sudden death. In contrast, there is a lack of evidence that calcium channel blockers are disease-modifying and their therapeutic role will be probably reduced in the near future. Long-acting nitrates are used on an empirical basis as randomised controlled trials are lacking, and are therefore expected to be replaced by the newer antianginal treatments.

Ranolazine, ivabradine or nicorandil are already indicated as second line options in patients inadequately controlled by first-line treatments or in whom such treatments are contraindicated or not tolerated. Ranolazine and ivabradine, which are associated with very low rates of side effects, will have increased roles in the management of stable angina patients that are older and with co-morbidities.

\section{Conclusion}

Chronic stable angina is still a major therapeutic challenge. Despite the UK guidelines in 2011, a number of unresolved issues remain, specifically disparities in the management of the older patient with multiple co-morbidities. It is expected that the newer anti-anginal treatments ranolazine and ivabradine will play an increasing role in the future clinical pathways for the management of stable angina.

\section{References}

1. Daly CA, Clemens F, Sendon JL, Tavazzi L, Boersma E, et al. (2005) The initial management of stable angina in Europe, from the Euro Heart Survey: a description of pharmacological management and revascularization strategies initiated within the first month of presentation to a cardiologist in the Euro Heart Survey of Stable Angina. Eur Heart J 26: 1011-1022.

${ }^{*}$ Corresponding author: Francesco Pelliccia, Department of Heart and Great Vessels, Sapienza University, Italy, E-mail: f. pelliccia@mclink. it

Received November 17, 2012; Accepted November 18, 2012; Published November 20, 2012

Citation: Pelliccia F (2012) Optimal Medical Therapy of Chronic Stable Angina: Current Guidelines and Future Perspectives. Cardiol Pharmacol 1:e102. doi:10.4172/2329-6607.1000e102

Copyright: (C) 2012 Pelliccia F. This is an open-access article distributed under the terms of the Creative Commons Attribution License, which permits unrestricted use, distribution, and reproduction in any medium, provided the original author and source are credited. 
Citation: Pelliccia F (2012) Optimal Medical Therapy of Chronic Stable Angina: Current Guidelines and Future Perspectives. Cardiol Pharmacol 1:e102. doi:10.4172/2329-6607.1000e102

Page 2 of 2

2. Fox K, Garcia MA, Ardissino D, Buszman P, Camici PG, et al. (2006) Guidelines on the management of stable angina pectoris: executive summary: The Task Force on the Management of Stable Angina Pectoris of the European Society of Cardiology. Eur Heart J 27: 1341-1381.

3. Fraker TD Jr, Finn SD, Gibbons RJ, Abrams J, Daley J, et al. (2007) 2007 chronic angina focused update of the ACC/AHA 2002 guidelines for the management of patients with chronic stable angina: a report of the American College of Cardiology/ American Heart Association Task Force on Practice Guidelines Writing Group to develop the focused update of the 2002 guidelines for the management of patients with chronic stable angina. J Am Coll Cordiol 50: 2264-2274.

4. http://www.nice.org.uk/CG126

5. Pepine CJ, Handberg EM, Cooper-DeHoff RM, Marks RG, Kowey P, et al (2003) A calcium antagonist vs a non-calcium antagonist hypertension treatment strategy for patients with coronary artery disease. The International Verapamil-
Trandolapril Study (INVEST): a randomized controlled trial. JAMA 290: 28052816.

6. Swedberg K, Komajda M, Bohm M, Borer JS, Ford I, et al. (2010) Ivabradine and outcomes in chronic heart failure (SHIFT): a randomised placebo-controlled study. Lancet 376: 875-885.

7. Conti CR (2011) Ranolazine and Silent ischemia. J Am Coll Cardiol 58: 1083.

8. Pelliccia F, Pasceri V, Marazzi G, Rosano G, Greco C, et al. (2012) A Pilot randomized study of ranolazine for reduction of myocardial damage during elective percutaneous coronary intervention. Am Heart J 163: 1019-1023.

9. Sakata Y, Nakatani D, Shimizu M, Suna S, Usami M, et al. (2012) Oral treatment with nicorandil at discharge is associated with reduced mortality after acute myocardial infarction. J Cardiol 59: 14-21. 